\title{
Decorating Microbially Produced Protein Nanowires with Peptide Ligands
}

2

3 Toshiyuki Ueki ${ }^{1,2}$, David J.F. Walker ${ }^{1,2}$, Pier-Luc Tremblay ${ }^{1,3}$, Kelly P. Nevin ${ }^{1}$, Joy E. Ward ${ }^{1}$,

4 Trevor L. Woodard ${ }^{1}$, Stephen S. Nonnenmann ${ }^{2,4}$, and Derek R. Lovley ${ }^{1,2 *}$

$6 \quad{ }^{1}$ Department of Microbiology, University of Massachusetts-Amherst, Amherst, MA, USA

$7 \quad{ }^{2}$ Institute for Applied Life Sciences, University of Massachusetts-Amherst

$8 \quad{ }^{3}$ Current address: School of Chemistry, Chemical Engineering and Life Science, Wuhan

$9 \quad$ University of Technology, Wuhan 430070, PR China

$10{ }^{4}$ Department of Mechanical and Industrial Engineering, University of Massachusetts-Amherst 


\section{Abstract}

13 The potential applications of electrically conductive protein nanowires (e-PNs) harvested from

14 Geobacter sulfurreducens might be greatly expanded if the outer surface of the wires could be

15 modified to confer novel sensing capabilities or to enhance binding to other materials. We

16 developed a simple strategy for functionalizing e-PNs with surface-exposed peptide ligands. The

17 G. sulfurreducens gene for the monomer that assembles into e-PNs was modified to add known

18 peptide ligands at the carboxyl terminus of the monomer. Strains of G. sulfurreducens were

19 constructed that fabricated synthetic e-PNs with a six-histidine 'His-tag' or both the His-tag and

20 a nine-peptide 'HA-tag' exposed on the outer surface. Addition of the peptide ligands did not

21 diminish e-PN conductivity. The abundance of HA-tag in e-PNs was controlled by placing

22 expression of the gene for the synthetic monomer with the HA-tag under transcriptional

23 regulation. These studies suggest broad possibilities for tailoring e-PN properties for diverse

24 applications.

26 Keywords: bioelectronic materials, e-biologics, pili, Geobacter, sustainable electronics 


\section{Introduction}

Microbially produced, electrically conductive protein nanowires (e-PNs) possess

31 properties, and possibilities for functionalization, not found in other electronic nanowire

32 materials ${ }^{1-5}$. Diverse microorganisms in both the Bacteria ${ }^{6-10}$ and Archaea ${ }^{11}$ produce e-PNs.

33 Functional analysis of Geobacter e-PNs have demonstrated that, when attached to cells, they

34 serve as conduits for long-range electron exchange with other cells or minerals ${ }^{1,12}$. The

35 biological roles of other microbial e-PNs have yet to be experimentally verified.

The most intensively studied e-PNs are the electrically conductive pili of the microorganism Geobacter sulfurreducens ${ }^{6,13-18}$. G. sulfurreducens assembles the thin (3 nm),

39 peptide ${ }^{6,16}$. Even though they are comprised of protein, the e-PNs produced with $G$.

40 sulfurreducens are remarkably robust. They retain function under conditions required for the

41 fabrication of electronic materials, including stability in a range of organic solvents and

42 temperatures greater than $100^{\circ} \mathrm{C}{ }^{1,19}$. The G. sulfurreducens e-PNs are produced from renewable

43 feedstocks. No harsh chemicals are required for e-PN production and there are no toxic

44 components in the final product. Unlike silicon nanowires, e-PNs do not dissolve in water or

45 bodily fluids, a distinct advantage for wearable and environmental electronic sensor applications,

46 as well as implantable electronics. e-PNs have evolved for making cell-to-cell electrical

47 connections ${ }^{1,12}$, suggesting they may be more biocompatible than other nanowire materials. The

48 dramatic change in e-PN conductivity in response to $\mathrm{pH}^{13,17}$ suggests that they may be readily

49 adapted for diverse sensor functions ${ }^{2}$.

Fabrication of e-PNs with G. sulfurreducens offers tight, reproducible, and consistent

51 control of nanowire structure and electronic properties, combined with the potential for broad 
52 possibilities in the design of novel e-PNs through genetic modification of the monomer peptide ${ }^{2}$.

53 For example, the conductivity of e-PNs produced with G. sulfurreducens have been tuned over

54 six orders of magnitude (ca. $1 \mathrm{mS} / \mathrm{cm}$ to $1 \mathrm{kS} / \mathrm{cm}$ ) ${ }^{17,18}$ by genetically manipulating the

55 abundance of aromatic amino acids in the monomer peptide.

We considered that the potential to genetically modify e-PN structure might make it

57 feasible to decorate the outer surface of e-PNs with short peptides. The design of synthetic $E$.

58 coli curli fibers with peptides that confer novel functions has greatly expanded their potential

59 applications ${ }^{20,21}$. However, curli fiber structure differs significantly from e-PN structure and,

60 unlike e-PNs, curli fibers are not intrinsically conductive. Curli fibers self-assemble outside the

61 cell $^{20,21}$, whereas the pilin monomers of $G$. sulfurreducens e-PNs are assembled into filaments

62 through a complex intracellular process ${ }^{22}$.

63 Modeling of the G. sulfurreducens e-PN structure predicted that amino acids at the

64 carboxyl terminal of the pilin monomer are likely to be exposed on the outer surface of the e-PNs

$65{ }^{16}$. This suggested that peptides might be added at the carboxyl terminus without destroying the

66 conductive properties of the e-PNs. If so, peptides could be introduced to confer new e-PN

67 functionalities for sensing applications, to facilitate nanowire alignment, and to link e-PNs with

68 other materials.

69 Therefore, we examined whether G. sulfurreducens would express e-PNs containing

70 monomers in which peptide ligands were added to the carboxyl terminus; whether the peptide

71 ligands introduced would be accessible on the outer surface of the e-PNs; and the potential

72 impact of the added peptide ligands on e-PN conductivity. The results suggest that $G$.

73 sulfurreducens e-PNs can be decorated with a diversity of outer surface peptide ligands to

74 introduce new binding properties. 


\section{Results and Discussion}

\section{Wires decorated with a six-histidine ligand}

77 In order to evaluate the possibility of displaying peptide ligands on the outer surface of e-

78 PNs, the wild-type G. sulfurreducens gene for the pilin monomer (PilA) was modified

79 (Supplementary Figure 1) to encode six histidines (i.e. a 'His-tag') at the carboxyl end (Figure

80 1a). This synthetic gene was inserted into the chromosome of G. sulfurreducens strain KN400

81 (Figure 1b), along with the gene for the protein $\mathrm{Spc}$ that is required for pilin monomer stability

$82{ }^{23}$. The resultant strain, which contained genes for the wild-type PilA as well as the histidine-

83 modified PilA pilin monomer (PilA-6His), was designated strain PilA-WT/PilA-6His. Western

84 analysis with anti-6His antibody of cell lysates of strain PilA-WT/PilA-6His separated with

85 SDS-PAGE revealed a single protein band at the molecular weight expected for the PilA-6His

86 monomer (Figure 1c). There was no corresponding band in lysates of wild-type cells. Western

87 analysis with antibody that detected wild-type PilA detected a single band in wild-type cell

88 lysates and two bands in lysates of strain PilA-WT/PilA-6His (Figure 1c). The additional band in

89 the strain PilA-WT/PilA-6His lysate was positioned at the higher molecular weight position

90 detected with the anti-6His antibody. 
a

$\begin{array}{lllllll}1 & 11 & 21 & 31 & 41 & 51 & 61\end{array}$

Wild-Type PiIA

FTLIELLIVVAIIGILAAIAIPQFSAYRVKAYNSAASSDLRNLKTALESAFADDQTYPPES

PilA-6His

FTLIELLIVVAIIGILAAIAIPQFSAYRVKAYNSAASSDLRNLKTALESAFADDQTYPPESHHHHHH

b

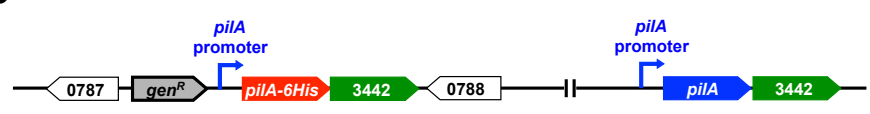

C

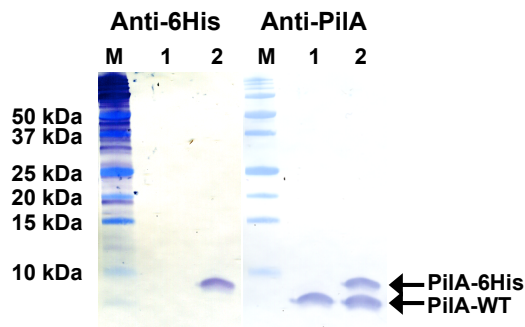

Figure 1. Construction of G. sulfurreducens strain PilA-WT/PilA-6His and expression of pilin monomers. (a) Amino acid sequence of the wild-type PilA and PilA-6His with the added histidines highlighted in red. (b) Location of wild-type and pilA-6His genes on the chromosome. Numbers are gene numbers from the genome sequence (NCBI, NC_017454.1). gen $^{R}$ is the gentamicin resistance gene. (c) Western blot analysis for the wild-type (lane 1) and PilA-WT/PilA-6His (lane 2) strains. Lane $M$ is molecular weight standard markers. Headings designate the antibody employed.

103 secondary antibody conjugated with gold revealed abundant His-tag loci along the wires that

104 were accessible to the antibody (Figure $2 \mathrm{a}, \mathrm{b}$ ). There was no gold labeling of wild-type cells

105 (Supplemental Figure 2).

106 When strain PilA-WT/PilA-6His cells were treated with a $\mathrm{Ni}^{2+}$-NTA-gold reagent

107 designed to label His-tags the gold nanoparticles were specifically localized along the wires 
108 (Figure $2 \mathrm{c}, \mathrm{d}, \mathrm{e})$. Wild-type cells were not labeled (Supplemental Figure 3). These results

109 demonstrate that the His-tag ligand was accessible on the outer surface of the wires.
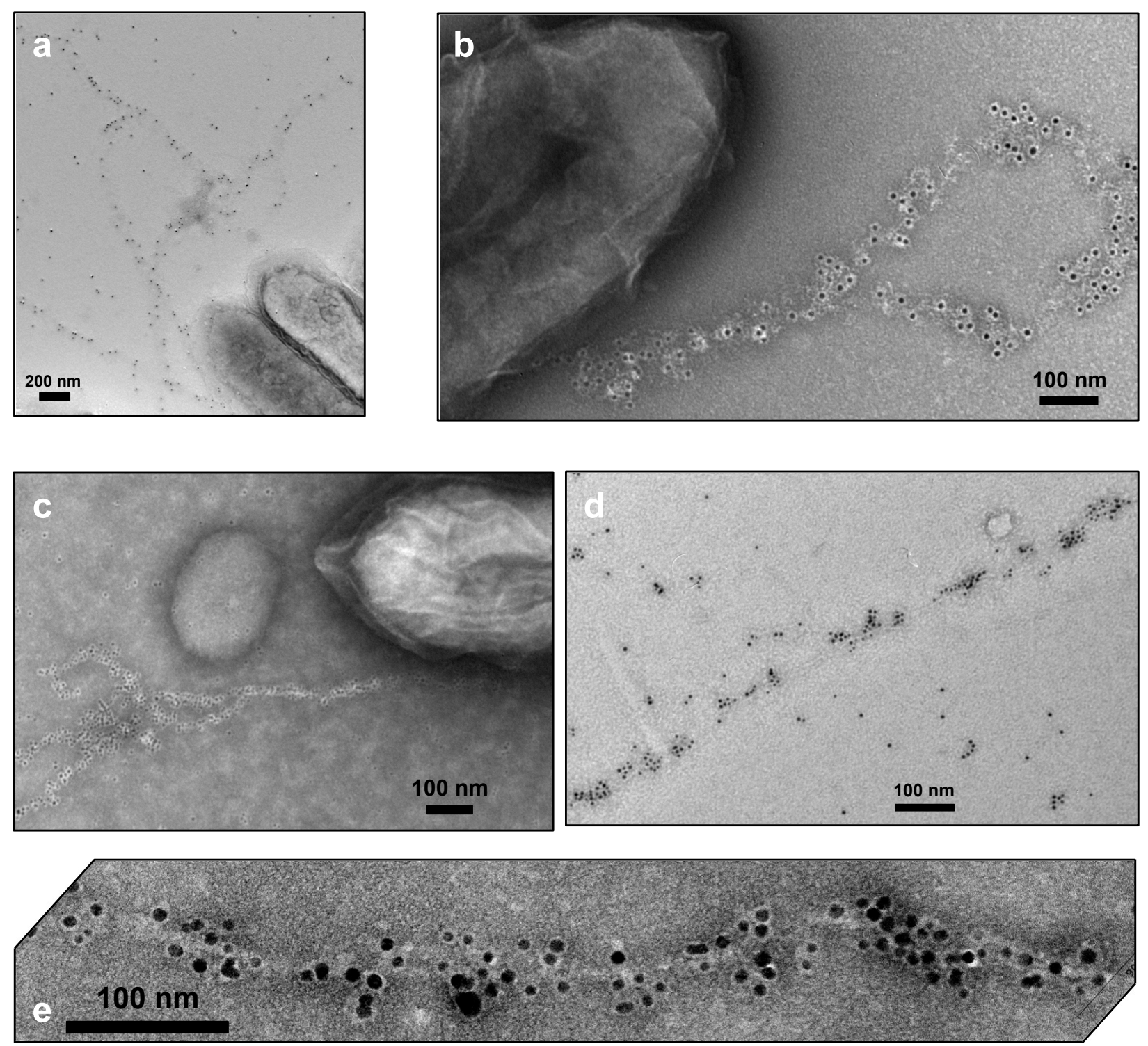

Figure 2. Transmission electron micrographs of immunogold $(\mathrm{a}, \mathrm{b})$ and $\mathrm{Ni}^{2+}$ -

113 NTA-gold (c, d, e) labeling of the 6His-tag incorporated into e-PNs of $G$.

114 sulfurreducens strain PilA-WT/PilA-6His. 
117 are electrically conductive ${ }^{14,24,25}$. G. sulfurreducens strain PilA-WT/PilA-6His produced

118 maximum currents comparable to the wild-type strain with just a slightly longer lag in the

119 initiation of current production (Figure 3). This result suggested that introducing the His-tag did 120 not substantially decrease pili conductivity.

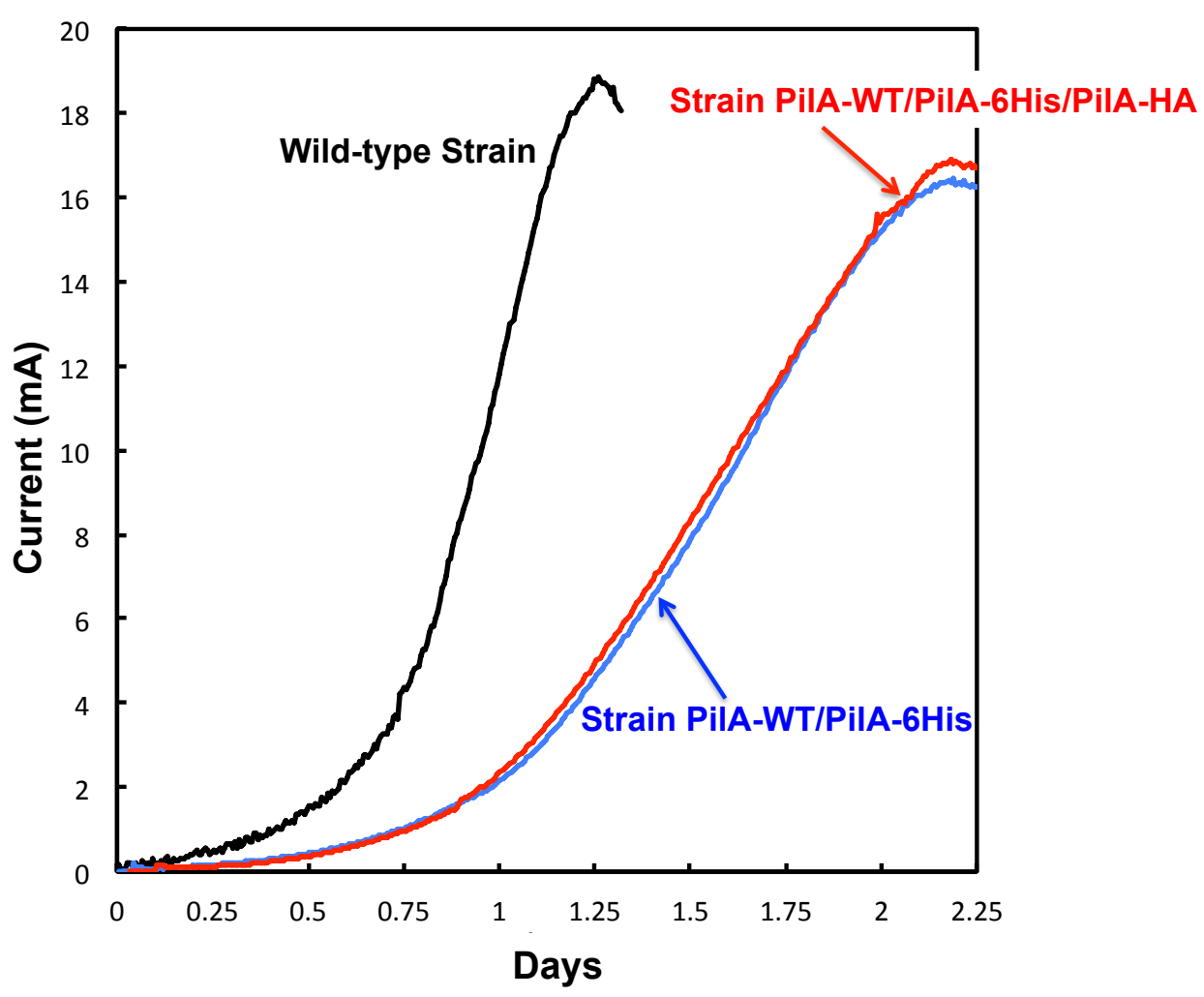

126 force microscopy (c-AFM), as previously described ${ }^{11}$. The wires were readily identified in

127 topographic imaging in contact mode and had a diameter of $3.1+0.3 \mathrm{~nm}($ mean + standard

128 deviation; $n=18,6$ points on 3 wires). The conductive tip was translated to the top of the wire, 
129 and the point-mode current-voltage response (I-V) spectroscopy revealed a conductance of $7.2+$

$1301.5 \mathrm{nS}($ mean + standard deviation; $\mathrm{n}=9)$ under a load force setpoint $(1 \mathrm{nN})$ (Figure 4,

131 Supplemental Figure 4). This is slightly higher than the previously observed ${ }^{11}$ conductance of

$1324.5+0.3 \mathrm{nS}$ for e-PNs comprised solely of the wild-type monomer and much higher than the

133 previously reported ${ }^{11}$ conductance of the e-PNs from strain G. sulfurreducens strain Aro-5,

134 which lacks key aromatic amino acids required for high conductivity.

a

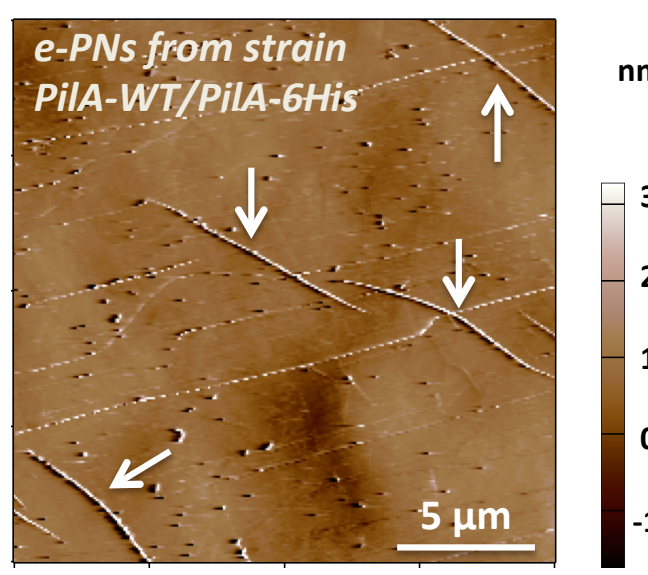

b
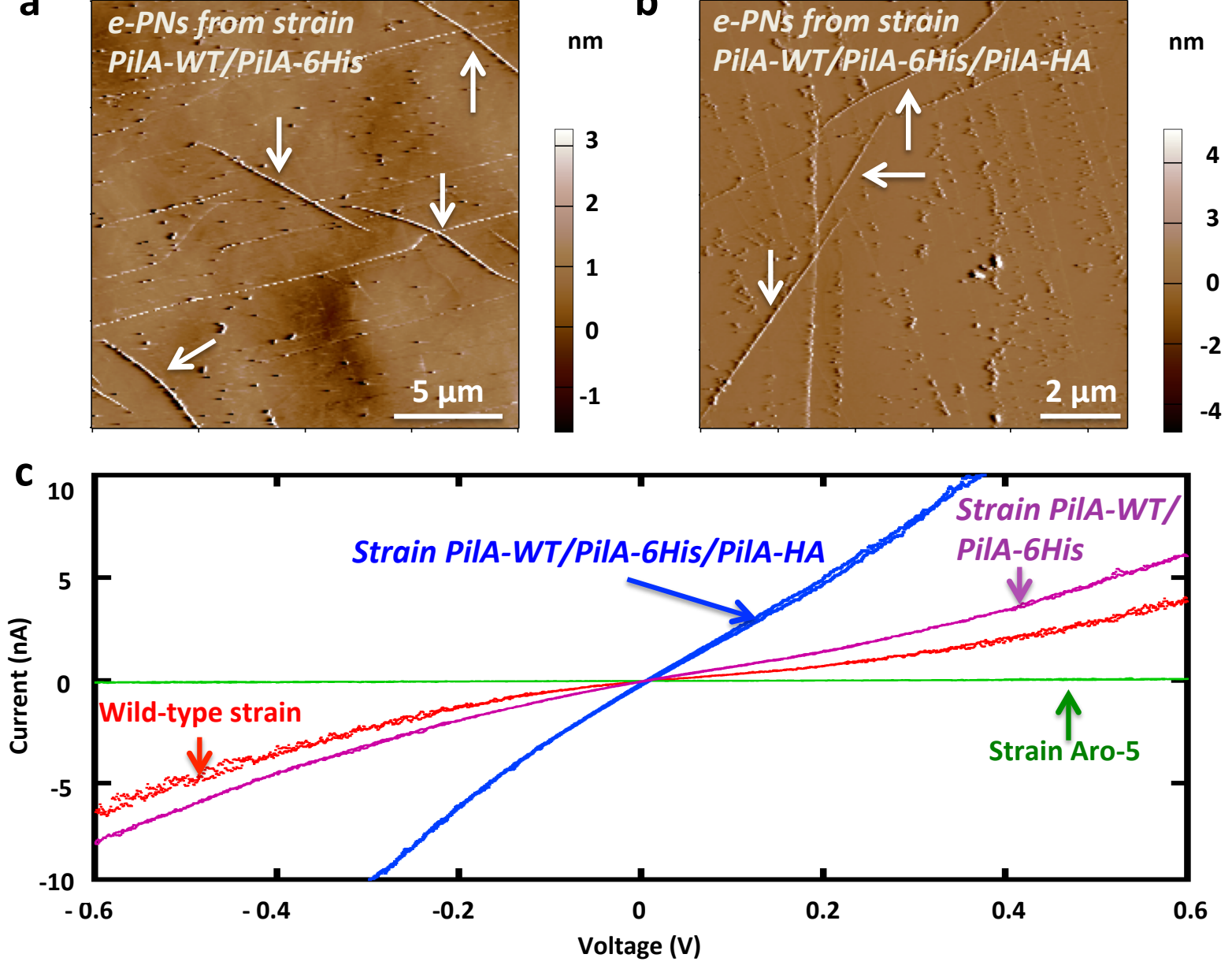

Figure 4. Current-voltage response of synthetic e-PNs decorated with peptide ligands. Representative contact-mode topographic imaging of e-PNs from (a) $G$. sulfurreducens strain PilA-WT/PilA-6His and (b) strain PilA-WT/PilA- 

published data ${ }^{11}$ on e-PNs from the wild-type strain of G. sulfurreducens and strain Aro-5, which expresses poorly conductive pili.

\section{Wires decorated with two different peptide ligands}

In order to determine whether two peptide ligands with different functions could be

147 displayed on one e-PN, a gene (Supplemental Figure 1) encoding the previously described ${ }^{26}$

148 nine-peptide 'HA-tag' (YPYDVPDYA) at the carboxyl end of the wild-type PilA pilin monomer

149 (Figure 5a) was incorporated into the chromosome along with the PilA-6His and wild-type (WT)

150 genes (Figure 5b). The gene for the PilA with the HA-tag (PilA-HA) was located downstream of

151 the IPTG-inducible lac promoter/operator in order to provide the option of controlling the

152 stoichiometry of incorporation of the PilA-HA monomer in the e-PNs (Figure 5b). This strain

153 was designated G. sulfurreducens strain PilA-WT/PilA-6His/PilA-HA. Western blot analysis

154 demonstrated that, in the presence of $1 \mathrm{mM}$ IPTG, monomers of WT-PilA, PilA-6His, and PilA-

155 HA were expressed in this strain (Figure 5c).

a

PilA-HA

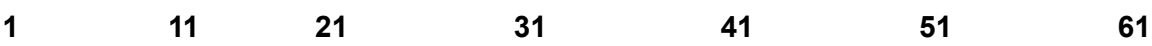

FTLIELLIVVAIIGILAAIAIPQFSAYRVKAYNSAASSDLRNLKTALESAFADDQTYPPESYPYDVPDYA

b

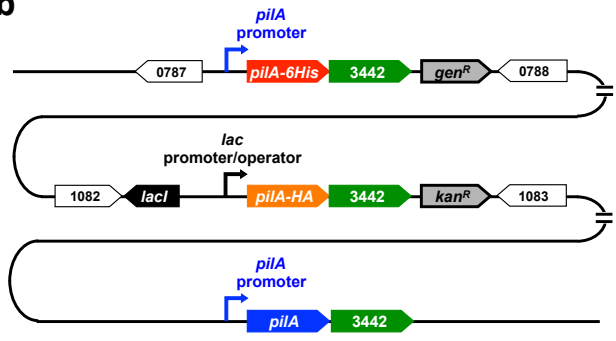

c

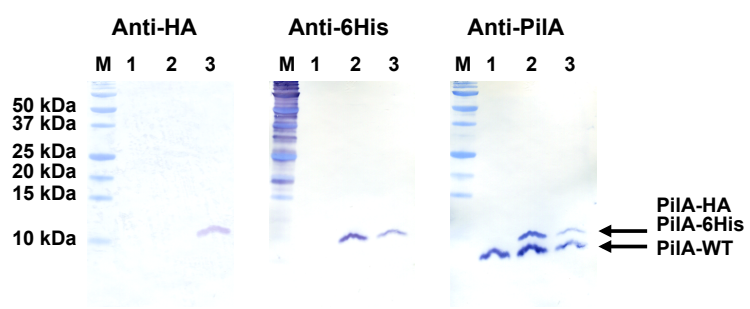


Figure 5. Construction of G. sulfurreducens strain PilA-WT/PilA-6His/PilA-HA and expression of pilin monomers. (a) Amino acid sequence of the PilA-HA with the HA tag highlighted in orange. (b) Location of wild-type, pilA-6His, and pilA$H A$ genes on the chromosome. lacI is the Lac repressor gene. $k_{a n}^{R}$ is the kanamycin resistance gene. (e) Western blot analysis of cells lysates of the wildtype (lane 1), PilA-WT/PilA-6His (lane 2), and PilA-WT/PilA-6His/PilA-HA (lane 3) strains. Lane $M$ is molecular weight standard markers. Headings Immunogold labeling for just the His-tag (Figure 6a,b) or the HA-tag (Figure 6c,d)

168 HA grown in the presence of $1 \mathrm{mM}$ IPTG. Dual labeling with secondary antibodies with

169 different size gold particles demonstrated that both tags were present in the same e-PNs (Figure 6

170 e,f). e-PNs of strain PilA-WT/PilA-6His cells were not immunogold labeled with the anti-HA

171 antibody (Supplementary Figure 5). The current production of strain PilA-WT/PilA-6His/PilA-

172 HA was similar to that of strain PilA-WT/PilA-6His, indicating the addition of the peptide tag

173 did not significantly diminish pili conductivity (Figure 3). In fact, analysis of individual e-PNs of

174 strain PilA-WT/PilA-6His/PilA-HA (Figure 4, Supplemental Figure 6) yielded higher currents at

175 equivalent applied voltages than observed with the e-PNs with just the His-tag, with an estimated

176 conductance of $27.2 \pm 1.0 \mathrm{nS}(\mathrm{n}=9)$. A potential explanation for the higher conductance is that

177 the HA-tag contains multiple aromatic amino acids, which may promote electron transport. 

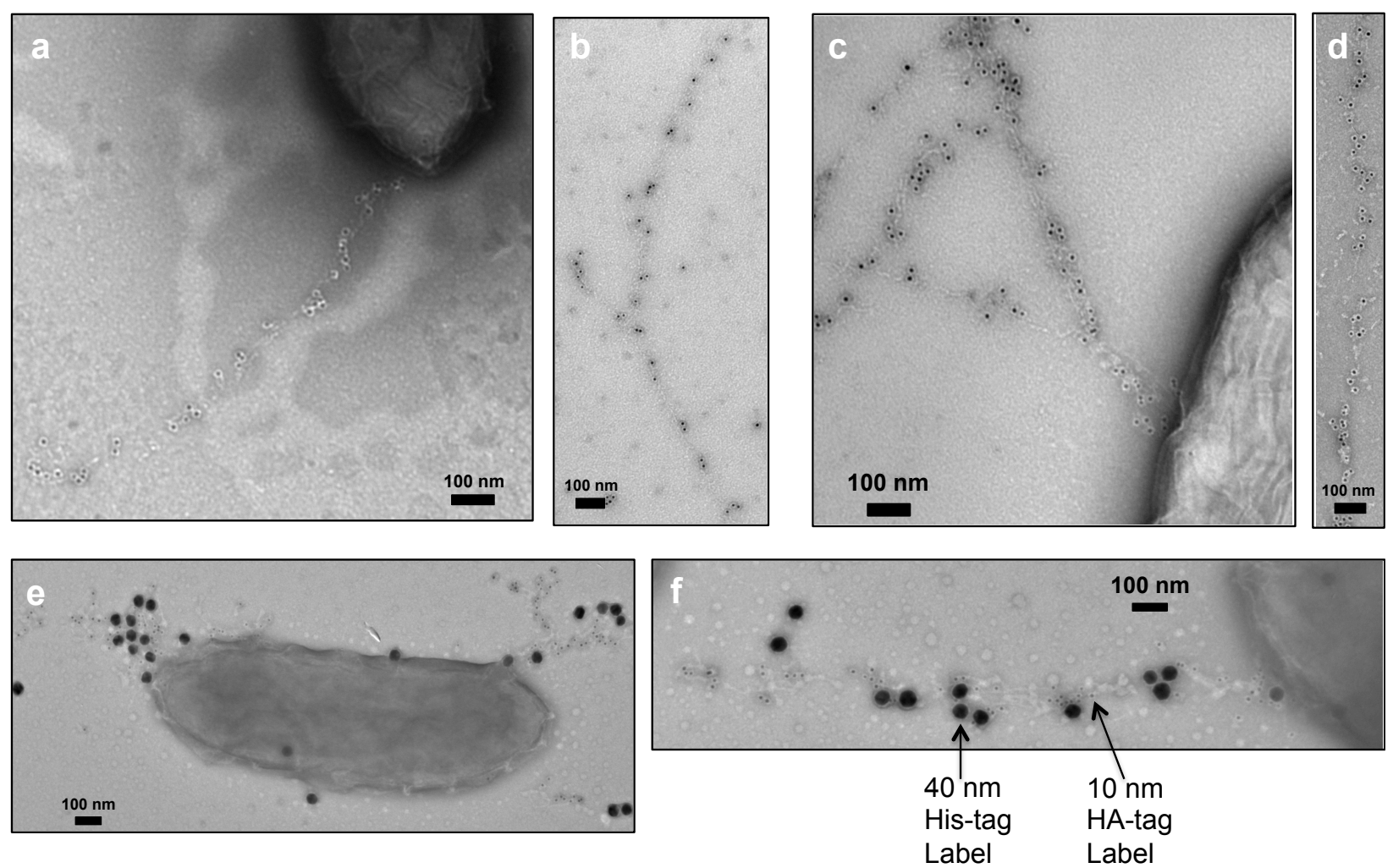

Figure 6. Transmission electron micrographs of immunogold labeling $G$. sulfurreducens strain PilA-WT/PilA-6His/PilA-HA for 6His-tag (a, b), HA-tag (c,

d), and both tags (e, f). Gold nanoparticles were $10 \mathrm{~nm}$ diameter throughout with the exception of the dual labeling in panels e and $\mathrm{f}$ in which the 6His-tag was labeled with $40 \mathrm{~nm}$ diameter gold.

186 of the IPTG inducer (Figure 7a). However, the concentration of PilA-HA monomer in the cells

187 was greater with added IPTG (Figure 7a). Increased pools of PilA-HA were associated with e-

188 PNs that labeled more heavily with immunogold labeling for the HA-tag (Figure 7b,c,d). These

189 results demonstrate that it is possible to control the abundance of a specific peptide ligand 
190 displayed on e-PNs with transcriptional control of the expression of the monomer with that

191 ligand.

a

Anti-HA

\section{$\begin{array}{lllll}M & 0 & 0.01 & 1 & \text { IPTG } \\ (\mathrm{mM})\end{array}$}

$50 \mathrm{kDa}$

$25 \mathrm{kDa}$

$20 \mathrm{kDa}$

$15 \mathrm{kDa}$

$10 \mathrm{kDa}$
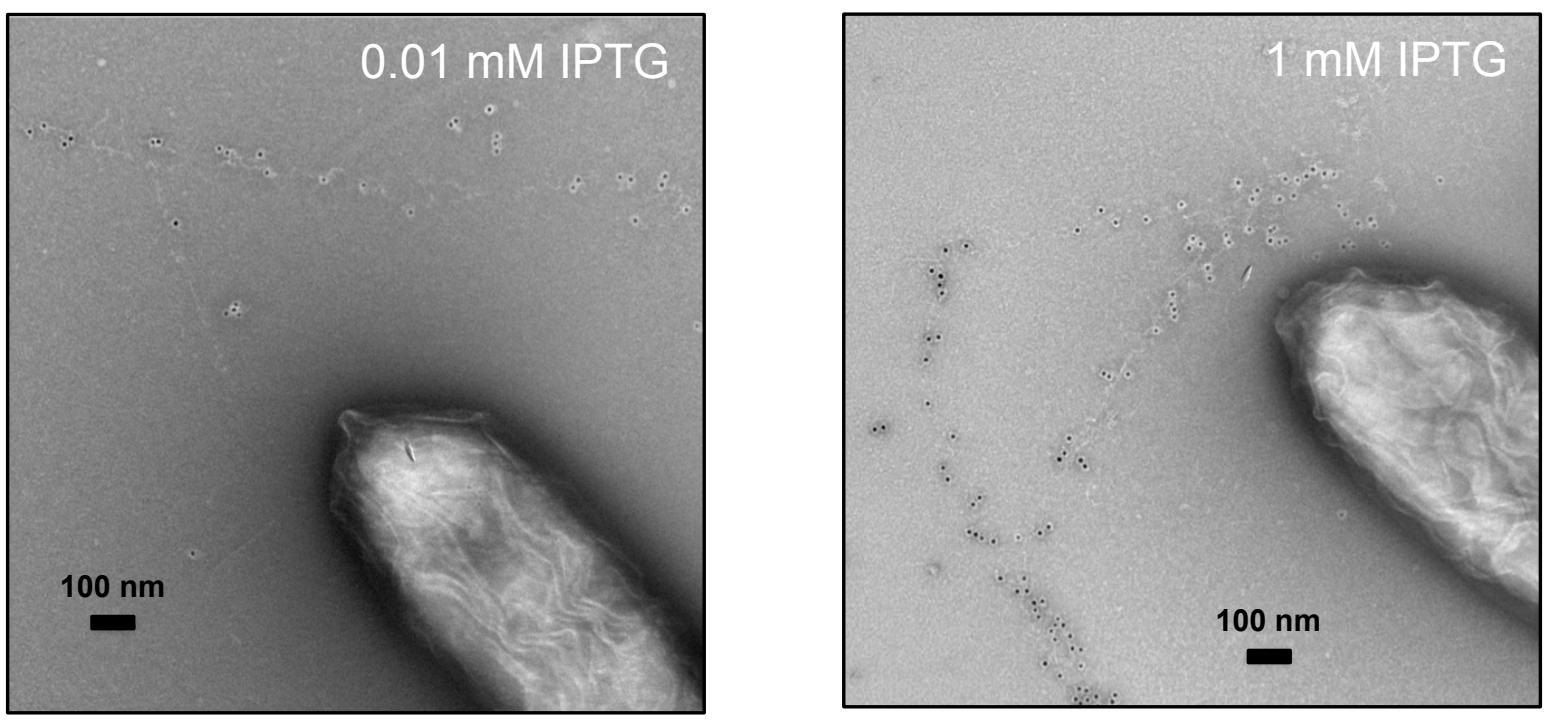

Figure 7. Transcriptional control of PilA-HA expression and incorporation into e-

PNs in strain PilA-WT/PilA-6His/PilA-HA evaluated with anti-HA antibody. (a)

Western blot analysis of cell lysates. Lane $\mathrm{M}$ is standard markers. (b, c, d)

Immunogold labeling of HA-tag in e-PNs during growth in the presence of the 


\section{Concluding Comments}

These results demonstrate that e-PNs produced with G. sulfurreducens can be decorated

201 with one or more peptide ligands while maintaining, or possibly increasing, their conductivity.

202 The stoichiometry of ligand density can be controlled with transcriptional regulation. These

203 capabilities greatly expand the potential applications of e-PNs in electronic devices and for the

204 fabrication of electrically conductive composite materials.

For example, sensors developed from other nanowire materials show promise for

206 providing highly sensitive and specific, real-time electrical response for detection of diverse

207 chemicals and biologics ${ }^{27-29}$. Analytes of interest are detected as a change in nanowire

208 conductivity that results from changes in $\mathrm{pH}$ associated with the activity of enzymes

209 incorporated into the sensors, or binding of analytes to nanowires functionalized with antibodies,

210 peptides, or other ligands. The conductivity of G. sulfurreducens e-PNs has already been shown

211 to be highly responsive to $\mathrm{pH}^{13,17}$. Short peptides for binding enzymes and antibodies ${ }^{30-35}$

212 displayed on the outer-surface of e-PNs could be an effective method for functionalizing e-PN-

213 based sensors. Furthermore, peptides can be designed to function as ligands for a wide diversity

214 of chemical and biological analytes or to enhance attachment to cells ${ }^{36-38}$. Thus, the simplicity of

215 modifying the peptides displayed on e-PNs, and controlling the abundance of peptide display,

216 could provide unprecedented flexibility in nanowire sensor design not readily achieved with

217 other nanowire materials. In a similar manner, modifying the surface chemistry of e-PNs with

218 short peptides or unnatural amino acids may enable chemical linkages with polymers or enhance

219 binding to materials to aid in e-PN alignment in electronic devices ${ }^{1}$.

220 The studies described here demonstrate that peptides of up to 9 amino acids can be added

221 to the 61 amino acid monomer backbone of G. sulfurreducens e-PNs. However, it may be 
222 possible to decorate e-PNs with much larger peptides because the monomers of other conductive

223 pili have an N-terminal end homologous to the G. sulfurreducens monomer, but are comprised of

224 over 100 amino acids ${ }^{10,39}$. These broad possibilities for modifying e-PNs with peptides coupled

225 with the advantages of e-PNs as a 'green' sustainable material suggest that further investigation

226 into the development of e-PN-based electronic devices and materials is warranted.

Materials and Methods

\section{Strains and growth conditions}

231 medium with acetate as the electron donor and fumarate as the electron acceptor as previously

232 described $^{40}$, unless otherwise described. Escherichia coli was cultivated with Luria-Bertani

233 medium with or without antibiotics ${ }^{41}$.

\section{Construction of G. sulfurreducens PilA-WT/PilA-6His strain}

236 KN400 ${ }^{42}$. A gene for PilA-6His, the gene KN400_3442, which is located downstream of the

237 pilA gene (KN400_1523) on the chromosome, and the putative transcription terminator were

238 integrated at a non-coding region between KN400_0788 and KN400_0787 in the chromosome

239 (Figure 1b). The primer pair upKpnI (CTAGGTACCGTGGTGGACCCCCTTACCGGT)/upSpeI

240 (CGAACTAGTTGTGACCGCTGCCGGCTCCG) was used to amplify by PCR ca. 550 bp of

241 KN400_0788 upstream of the integration site with the genomic DNA as template. This PCR

242 product was digested with KpnI/SpeI and ligated with the vector $\mathrm{pCR} 2.1 \mathrm{Gm}^{\mathrm{r}}$ loxP ${ }^{43}$, resulting in

243 pCR2.1UP-Gm ${ }^{\mathrm{r}}$ loxP. The 6His tag was fused to the C-terminal end of PilA by PCR with the

244 primer pair pilAdnNotI (CACGCGGCCGCAAGAGGAGCCAGTGACGAAAATC)/pilACHis 

construction of pilA-6His-KN400_3442, two PCR products were generated before being

247 combined by recombinant PCR. pilA-6His was amplified with the primer pair

248 pilAdnNotI/pilAHisrecup

249 (CTCCAGTATGTATTTAATCAATTAGTGGTGGTGGTGGTGGTG) while KN400_3442 was 250 amplified with the primer pair pilAHisrecdn

(CACCACCACCACCACCACTAATTGATTAAATACATACTGGAG)/GSU1497XhoIAvrII

254 recombinant PCR. pilA-6His-KN400_3442 was cloned at NotI/XhoI sites in pCR2.1UP-Gm ${ }^{\mathrm{r}}$ loxP

255 downstream of $\mathrm{Gm}^{\mathrm{r}}$ lox $P$, resulting in pCR2.1UP-Gm ${ }^{\mathrm{r}}$ loxP-pilAHis-3442. The primer pair dnAvrII (CATCCTAGGAGGGCAGACATTGCGGAACGT)/dnXhoI

257 (CATCTCGAGCGGGTTCCGCTGCCGTCGTAC) was used to amplify by PCR ca. 530 bp of 258 KN400_0787 downstream of the integration site. This PCR product was cloned at AvrII/XhoI 259 sites in pCR2.1UP-Gm ${ }^{\mathrm{r}}$ loxP-pilAHis-3442, resulting in pCR2.1UP-Gm ${ }^{\mathrm{r}}$ loxP-pilAHis-3442-DN.

260 The final plasmid was linearized with XhoI for transformation as previously described ${ }^{40}$.

261 Transformants were selected with the medium containing gentamicin $(20 \mu \mathrm{g} / \mathrm{ml})$ and were

262 verified by PCR.

\section{Construction of G. sulfurreducens PilA-WT/PilA-6His/PilA-HA strain}

G. sulfurreducens PilA-WT/PilA-6His/PilA-HA strain was constructed by introducing a 265 gene encoding PilA monomer with the HA tag (pilA-HA) together with the gene KN400_3442 in

266 the chromosome of the G. sulfurreducens PilA-WT/PilA-6His strain (Figure 5b). The pilA-HA 267 gene was amplified by PCR with a primer pair 

amplified by PCR with a primer pair

273 were connected by PCR with a primer pair cloned in the plasmid pKIkan, which is a derivative of pKIapr ${ }^{44}$ and has a kanamycin-resistance

277 gene instead of the apramycin-resistance gene. The sequences of KN400_1082 and 1083 used for

278 homologous recombination for introduction of pilA-HA/KN400_3442 are same as those of

279 GSU1106 and 1107 for homologous recombination sequences in pKIkan, respectively. The

280 plasmid thus constructed was linearized with XhoI for electroporation.

\section{Western blot analysis}

The wild-type, PilA-WT/PilA-6His, and PilA-WT/PilA-6His/PilA-HA strains were

283 grown with acetate and fumarate at $25^{\circ} \mathrm{C}$. IPTG was added at $1 \mathrm{mM}$ for the PilA-WT/PilA-

284 6His/PilA-HA strain with the exception of the study of the impact on IPTG concentrations on

285 incorporation of PilA-HA in filaments. Cell extracts were prepared with B-PER Complete

286 Bacterial Protein Extraction Reagent (Thermo Fisher Scientific) and the amount of protein was

287 measured with the Bradford Protein Assay (Bio-Rad) as instructed by the manufacturer. Cell

288 extracts were separated on 16.5\% Tris-Tricine gel (Bio-Rad). An anti-PilA antibody was

289 obtained against peptide, ESAFADDQTYPPES, corresponding to the C-terminal end of PilA

290 (New England Peptide). An anti-6His antibody (6x-His Tag Polyclonal Antibody) and an anti- 
291 HA antibody (HA Tag Polyclonal Antibody) were purchased from Invitrogen. Western blot

292 analysis was conducted as described previously ${ }^{45}$.

293 Immunogold labeling

294 The strains were grown with acetate and fumarate at $25^{\circ} \mathrm{C}$. The PilA-WT/PilA-6His/PilA-

295 HA strain was grown with $1 \mathrm{mM}$ IPTG unless otherwise specified. Immunogold labeling was

296 conducted as previously described ${ }^{45}$. For immunogold labeling of just one type of ligand, the $6 x-$

297 His Tag Polyclonal Antibody or HA Tag Polyclonal Antibody was the primary antibody and the

298 anti-rabbit IgG-gold (10 nm) antibody (Sigma-Aldrich) was the secondary. Dual immunogold

299 labeling was conducted with 6x-His Tag Monoclonal Antibody (Invitrogen) and the HA Tag

300 Polyclonal Antibody as primary antibodies and an anti-mouse IgG-gold (40 nm) antibody (40 nm

301 Goat Anti-Mouse IgG gold conjugate, Expedeon) and the anti-rabbit IgG-gold (10 nm) antibody

302 as secondary antibodies. Samples were examined with transmission electron microscopy as

303 described previously ${ }^{7}$.

$304 \mathrm{Ni}^{2+}$-binding assay

305 The wild-type and PilA-WT/PilA-6His strains were grown with acetate and fumarate at

$30625^{\circ} \mathrm{C} . \mathrm{Ni}^{2+}$-binding assay was conducted with Ni-NTA-Nanogold ( $\left.5 \mathrm{~nm}\right)$ (Nanoprobes). Seven $\mu 1$

307 of the culture was placed on a copper grid and incubated for $5 \mathrm{~min}$. The grid was floated upside

308 down in phosphate-buffered saline (PBS) for $5 \mathrm{~min}$, in PBS containing 3\% bovine serum

309 albumin (BSA) and $40 \mathrm{mM}$ imidazole for $15 \mathrm{~min}$, and in PBS containing 0.3\% BSA, $40 \mathrm{mM}$

310 imidazole, and the Ni-NTA-Nanogold for $30 \mathrm{~min}$ at room temperature. The grid was washed

311 with PBS containing $40 \mathrm{mM}$ imidazole three times and with water once. Samples were stained

312 with $2 \%$ uranyl acetate and examined by transmission electron microscopy as described

313 previously ${ }^{7}$. 


\section{Current production}

315

316

317

318

\section{9}

320

321

322

323

324

325

326

327

328

329

The capacity to produce current was determined in the two-chambered H-cell system with a continuous flow of medium with acetate $(10 \mathrm{mM})$ as the electron donor and graphite stick anode $\left(65 \mathrm{~cm}^{2}\right)$ poised at $300 \mathrm{mV}$ versus $\mathrm{Ag} / \mathrm{AgCl}$ as the electron acceptor as described previously ${ }^{46}$.

\section{Conductive atomic force microscopy (c-AFM) of individual e-PNs}

As previously described ${ }^{11}$, an aliquot $(100 \mu$ l) of cell culture was drop-cast onto highly oriented pyrolytic graphite (HOPG). Conductive atomic force microscopy was preformed using an Oxford Instruments/Asylum Research Cypher ES atomic force microscope with a Pt/Ir-coated Arrow-ContPT tip (NanoWorld AG, Neuchâtel, Switzerland). Topographic imaging was performed in contact mode with a force of $0.1 \mathrm{nN}$. Point-mode current-voltage response (I-V) spectroscopy was achieved by applying a $1 \mathrm{nN}$ force to the top of the wire and conducting quadruplicate voltage sweeps of -0.6 to $0.6 \mathrm{~V}$ at $0.99 \mathrm{~Hz}$. The voltage sweep was averaged for each of the I-V curves and conductance was calculated from the linear portion of the I-V curve (0.2 to $0.2 \mathrm{~V}$ ) (Supplemental Figure 4). Average conductance and standard deviation were calculated using 3 independent points on 3 independent e-PNs of each strain. Average height and standard deviation were calculated from 6 independent points on 3 independent e-PNs.

\section{References}

1. Lovley, D. R., Electrically conductive pili: biological function and potential applications in electronics. Curr. Opin. Electrochem. 2017, 4, 190-198.

2. Lovley, D. R., e-Biologics: Fabrication of sustainable electronics with 'green' biological materials. mBio 2017, 8, e00695-17.

3. Gutermann, T.; Gazit, E., Toward peptide-based bioelectronics: reductionist design of conductive pili mimetics. Bioelectronics Med. 2018, 1, 131-137. 
4. Creasey, R. C. G.; Mostert, A. B.; Nguyen, T. A. H.; Virdis, B.; Freguia, S.; Laycock, B., Microbial nanowires -electron transport and the role of synthetic analogues Acta Biomater. 2018, 69, 1-30.

5. Ing, N. L.; El-Naggar, M. Y.; Hochbaum, A. I., Going the distance: long-range conductivity in protein and peptide bioelectronic materials. J. Phys. Chem. B 2018, 122: 1040310423.

6. Reguera, G.; McCarthy, K. D.; Mehta, T.; Nicoll, J. S.; Tuominen, M. T.; Lovley, D. R., Extracellular electron transfer via microbial nanowires. Nature 2005, 435, 1098-1101.

7. Tan, Y.; Adhikari, R. Y.; Malvankar, N. S.; Ward, J. E.; Woodard, T. L.; Nevin, K. P.; Lovley, D. R., Expressing the Geobacter metallireducens PilA in Geobacter sulfurreducens yields pili with exceptional conductivity. mBio 2017, 8, e02203-16.

8. Holmes, D. E.; Dang, Y.; Walker, D. J. F.; Lovley, D. R., The electrically conductive pili of Geobacter species are a recently evolved feature for extracellular electron transfer. Microbial Genomics 2016, 2, doi: 10.1099/mgen.0.000072.

9. Sure, S.; Ackland, M. L.; Torriero, A. J.; Adholeya, A.; Kochar, M., Microbial nanowires: an electrifying tale. Microbiology 2016, 162, 2017-2028.

10. Walker, D. J. F.; Adhikari, R. Y.; Holmes, D. E.; Ward, J. E.; Woodard, T. L.; Nevin, K. P.; Lovley, D. R., Electrically conductive pili from genes of phylogenetically diverse microorganisms. ISME J. 2018, 12, 48-58.

11.Walker, D. J. F.; Martz, E.; Holmes, D. E.; Zhou, Z.; Nonnenmann, S. S.; Lovley, D. R., The archaellum of Methanospirillum hungatei is electrically conductive. mBio 2019, 10, (in press).

12. Lovley, D. R., Syntrophy goes electric: direct interspecies electron transfer. Ann. Rev. Microbiol. 2017, 71, 643-664.

13. Malvankar, N. S.; Vargas, M.; Nevin, K. P.; Franks, A. E.; Leang, C.; Kim, B.-C.; Inoue, K.; Mester, T.; Covalla, S. F.; Johnson, J. P.; Rotello, V. M.; Tuominen, M. T.; Lovley, D. R., Tunable metallic-like conductivity in nanostructured biofilms comprised of microbial nanowires. Nature Nanotechnology 2011, 6, 573-579.

14. Vargas, M.; Malvankar, N. S.; Tremblay, P.-L.; Leang, C.; Smith, J. A.; Patel, P.; Snoeyenbos-West, O.; Nevin, K. P.; Lovley, D. R., Aromatic amino acids required for pili conductivity and long-range extracellular electron transport in Geobacter sulfurreducens mBio 2013, 4, e00105-13. .

15. Malvankar, N. S.; Yalcin, S. E.; Tuominen, M. T.; Lovley, D. R., Visualization of charge propagation along individual pili proteins using ambient electrostatic force microscopy. Nature Nanotechnology 2014, 9, 1012-1017.

16. Malvankar, N. S.; Vargas, M.; Nevin, K. P.; Tremblay, P.-L.; Evans-Lutterodt, K.; Nykypanchuk, D.; Martz, E.; Tuominen, M. T.; Lovley, D. R., Structural basis for metallic-like conductivity in microbial nanowires. mBio 2015, 6, e00084-15.

17. Adhikari, R. Y.; Malvankar, N. S.; Tuominen, M. T.; Lovley, D. R., Conductivity of individual Geobacter pili. RSC Advances 2016, 6, 8354-8357.

18. Tan, Y.; Adhikari, R. Y.; Malvankar, N. S.; Pi, S.; Ward, J. E.; Woodard, T. L.; Nevin, K. P.; Xia, Q.; Tuominen, M. T.; Lovley, D. R., Synthetic biological protein nanowires with high conductivity. Small 2016, 12, 4481-4485.

19. Sun, Y.-L.; Tang, H.-.-Y.; Ribbe, A.; Duzhko, V.; Woodard, T. L.; Ward, J. E.; Nevin, K. P.; Nonnenmann, S.; Russell, T. P.; Emrick, T.; Lovley, D. R., Conductive composite 
materials fabricated with microbially produced protein nanowires. Small 2018, 14 , 1802624.

20. Wang, Y.; Pu, J.; An, B.; Lu, T. K.; Zhong, C., Emerging paradigms for synthetic design of functional amyloids. J. Mol. Biol. 2018, 430, 3720-3734. 21. Nguyen, P. Q.; Dorval Courchesne, N.-M.; Duraj-Thatte, A.; Praveschotinunt, P.; Joshi, N. S., Engineered living materials: prospects and challenges for using biological systems to direct the assembly if smart Materials. Adv. Materials 2018, 30, 1704847.

22. Hospenthal, M. K.; Costa, T. R. D.; Waksman, G., A comprehensive guide to pilus biogenesis in Gram-negative bacteria. Nature Reviews Microbiology 2017, 15, 365-379.

23. Liu, X.; Zhou, S.; Lovley, D. R., A pilin chaperone required for the expression of electrically conductive Geobacter sulfurreducens pili 2018, (manuscript submitted).

24. Liu, X.; Tremblay, P.-L.; Malvankar, N. S.; Nevin, K. P.; Lovley, D. R.; Vargas, M., A Geobacter sulfurreducens strain expressing Pseudomonas aeruginosa type IV pili localizes OmcS on pili but Is deficient in Fe(III) oxide reduction and current production. Appl. Environ. Microbiol. 2014, 80, 1219-1224.

25. Tan, Y.; Adhikari, R. Y.; Malvankar, N. S.; Ward, J. E.; Nevin, K. P.; Woodard, T. L.; Smith, J. A.; Snoeyenbos-West, O. L.; Franks, A. E.; Tuominen, M. T.; Lovley, D. R., The low conductivity of Geobacter uraniireducens pili suggests a diversity of extracellular electron transfer mechanisms in the genus Geobacter. Front. Microbiol. 2016, 7, 980 .

26. Field, J.; Nikawa, J.; Broek, D.; MacDonald, B.; Rodgers, L.; Wilson, I. A.; Lerner, R. A.; Wigler, M., Purification of a RAS-responsive adenylyl cyclase complex from Saccharomyces cerevisiae by use of an epitope addition method. Mol. Cell. Biol. 1988, 8, 2159-65.

27. Ahmad, R.; Mahmoudi, T.; Ahn, M. S.; Hahn, Y. B., Recent advances in nanowires-based $\mathrm{f}$ ield-effect transistors for biological sensor applications. Biosens. Bioelec. 2018, 100, 312325.

28. Zhang, A.; Lieber, C. M., Nano-Bioelectronics. Chem. Rev. 2016, 116, 215-57.

29. Zhu, C.; Yang, G.; Li, H.; Du, D.; Lin, Y., Electrochemical sensors and biosensors based on nanomaterials and nanostructures. Anal.Chem, 2015, 87, 230-49.

30. Lu, Y.; Huang, F.; Wang, J.; Xia, J., Affinity-guided covalent conjugation reactions based on PDZ-peptide and SH3-peptide interactions. Bioconjug. Chem. 2014, 25, 989-99.

31. Yan, X.; Zhou, H.; Zhang, J.; Shi, C.; Xie, X.; Wu, Y.; Tian, C.; Shen, Y.; Long, J., Molecular mechanism of inward rectifier potassium channel 2.3 regulation by taxinteracting protein-1. J, Mol. Biol. 2009, 392, 967-76.

32. Huang, J.; Nagy, S. S.; Koide, A.; Rock, R. S.; Koide, S., A peptide tag system for facile purification and single-molecule immobilization. Biochemistry 2009, 48, 11834-6.

33. Gaidamakova, E. K.; Backer, M. V.; Backer, J. M., Molecular vehicle for target-mediated delivery of therapeutics and diagnostics. $J$ Control Release 2001, 74, 341-7.

34. Zakeri, B.; Fierer, J. O.; Celik, E.; Chittock, E. C.; Schwarz-Linek, U.; Moy, V. T.; Howarth, M., Peptide tag forming a rapid covalent bond to a protein, through engineering a bacterial adhesin. Proc Natl Acad Sci U S A 2012, 109, E690-7.

35. Skerra, A.; Schmidt, T. G., Use of the Strep-Tag and streptavidin for detection and purification of recombinant proteins. Methods Enzymol. 2000, 326, 271-304.

36. Pavan, S.; Berti, F., Short peptides as biosensor transducers. Anal. Bioanal. Chem. 2012, 402. 37. Liu, Q.; Wang, J.; Boyd, B. J., Peptide-based biosensors. Talanta 2015, 136, 114-127. 
38. Chen, H.; Huang, J.; Palaniappan, A.; Wang, Y.; Liedberg, B.; Platt, M.; Tok, A. L. Y., A review on electronic bio-sensing approaches based on non-antibody recognition elements. Analyst 2016, 141, 2335.

39. Walker, D. J. F.; Nevin, K. P.; Nonnenmann, S. S.; Holmes, D. E.; Woodard, T. L.; Ward, J. E.; Rotaru, A.-E.; McInerney, M. J.; Lovley, D. R., Syntrophus conductive pili demonstrate that common hydrogen-donating syntrophs can have a direct electron transfer option bioRxiv 2018, https://www.biorxiv.org/content/early/2018/11/28/479683.

40. Coppi, M. V.; Leang, C.; Sandler, S. J.; Lovley, D. R., Development of a genetic system for Geobacter sulfurreducens. Appl. Environ. Microbiol. 2001, 67, 3180-3187.

41. Sambrook, J. a. R., D.W., Molecular cloning: a laboratory manual 3rd ed. Cold Spring Harbor: Cold Spring Harbor, NY, 2001.

42. Yi, H.; Nevin, K. P.; Kim, B.-C.; Franks, A. E.; Klimes, A.; Tender, L. M.; Lovley, D. R., Selection of a variant of Geobacter sulfurreducens with enhanced capacity for current production in microbial fuel cells. Biosensors Bioelectron. 2009, 24, 3498-3503.

43. Aklujkar, M.; Lovley, D. R., Interference with histidyl-tRNA synthetase by a CRISPR spacer sequence as a factor in the evolution of Pelobacter carbinolicus. BMC Evol Biol 2010, $10,230$.

44. Ueki, T.; DiDonato, L. N.; Lovley, D. R., Toward establishing minimum requirements for extracellular electron transfer in Geobacter sulfurreducens FEMS Microbiol. Lett. 2017, 364, fnx093.

45.Leang, C.; Qian, X.; Mester, T.; Lovley, D. R., Alignment of the $c$-type cytochrome OmcS along pili of Geobacter sulfurreducens. Appl Environ Microbiol 2010, 76, 4080-4084.

46. Nevin, K. P.; Kim, B.-C.; Glaven, R. H.; Johnson, J. P.; Woodard, T. L.; Methé, B. A.; DiDonato Jr, R. J.; Covalla, S. F.; Franks, A. E.; Liu, A.; Lovley, D. R., Anode biofilm transcriptomics reveals outer surface components essential for high current power production in Geobacter sulfurreducens fuel cells. PLoS ONE 2009, 4, e5628. 